\title{
Comparison of energy balance-related behaviours and measures of body composition between Turkish adolescents in Turkey and Turkish immigrant adolescents in the Netherlands
}

\author{
Gamze Akbulut 1,*, Mine Yildirim², Nevin Sanlier ${ }^{1}$, Maartie M van Stralen², \\ Nilufer Acar-Tek ${ }^{1}$, Saniye Bilici ${ }^{1}$, Johannes Brug ${ }^{3}$, Judith SB de Meii ${ }^{4}$, Makbule \\ Gezmen-Karadag ${ }^{1}$, Eda Koksal ${ }^{1}$, Anke Oenema ${ }^{5}$, Amika S Singh ${ }^{2}$, Saskia J te Velde ${ }^{3}$, \\ Hilal Yildiran ${ }^{1}$ and Mai JM Chinapaw ${ }^{2}$ \\ 'Department of Nutrition and Dietetics, Faculty of Health Sciences, Gazi University, Emniyet Mah., Muammer \\ Yasar Bostanci Cad No: 16, Besevler, Ankara, Turkey: ${ }^{2}$ Department of Public and Occupational Health and the \\ EMGO Institute for Health and Care Research, VU University Medical Center, Amsterdam, the Netherlands: \\ ${ }^{3}$ Department of Epidemiology and Biostatistics and the EMGO Institute for Health and Care Research, VU \\ University Medical Center, Amsterdam, the Netherlands: ${ }^{4}$ Department of Epidemiology, Documentation and \\ Health Promotion, Municipal Health Service of Amsterdam, Amsterdam, the Netherlands: ${ }^{5}$ Department of Health \\ Promotion, Maastricht University, Maastricht, the Netherlands
}

Submitted 17 December 2012: Final revision received 22 October 2013: Accepted 20 November 2013: First published online 16 December 2013

\begin{abstract}
Objective: To explore the influences of migration to a Western country on obesity and related risk factors by comparing measures of body composition and energy balance-related behaviours between Turkish adolescents in Turkey (TR-TR) and adolescents from Turkish immigrant ethnicity in the Netherlands (TR-NL).

Design: Cross-sectional survey or baseline intervention data from six Dutch school-based studies and one Turkish study.

Setting: Primary and secondary schools.

Subjects: A total of 915 (49\% girls; mean age $13 \cdot 1$ (SD 0 0 ) years) TR-TR adolescents and 433 (51\% girls; mean age $11 \cdot 7$ (SD $1 \cdot 3$ ) years) TR-NL adolescents were included. Outcome measures were self-reported sugar-containing beverage consumption, fruit and vegetable intake, screen time, physical activity, measured body height and weight, BMI, waist and hip circumferences, and skinfold thicknesses.

Results: Our data showed that more TR-NL adolescents were overweight $(31 \% v$. $26 \%)$ and obese $(9 \% v, 6 \%)$ and had significantly higher mean BMI $(21 \cdot 1 v$. $\left.20 \cdot 0 \mathrm{~kg} / \mathrm{m}^{2}\right)$, waist circumference $(72 \cdot 2 \mathrm{v} \cdot 71 \cdot 3 \mathrm{~cm})$ and suprailiac skinfold thickness $(19 \cdot 8 v \cdot 13 \cdot 1 \mathrm{~mm})$ than TR-TR adolescents. TR-NL adolescents reported significantly higher sugar-containing beverage consumption $(1173 v .115 \mathrm{ml} / \mathrm{d})$, less fruit and vegetable intake (295 v. $647 \mathrm{~g} / \mathrm{d})$, less screen time $(253 v .467 \mathrm{~min} / \mathrm{d})$ and higher physical activity levels (61 v. $27 \mathrm{~min} / \mathrm{d})$ than TR-TR adolescents.

Conclusions: Immigrant adolescents in the Netherlands were more often overweight and had a less favourable dietary pattern than their peers in Turkey, while their physical activity and screen time patterns were more favourable. These results suggest that adolescents from Turkish immigrant ethnicity in the Netherlands have adopted lifestyles towards the host culture.
\end{abstract}

Keywords
Overweight
Migrants
Adolescents
Energy balance-related behaviours

Overweight and obesity are major public health problems leading to an increased risk of non-communicable diseases $^{(1)}$. The prevalence of obesity and associated comorbidities among children and adolescents has risen worldwide throughout the past three decades ${ }^{(2)}$. It has been estimated that $10 \%$ of schoolchildren are overweight and a quarter of them are obese worldwide, whereas in Europe and America the prevalence is above
$20 \%{ }^{(3)}$. Obese children are not only at risk for becoming obese adults ${ }^{(4)}$, they are also more likely to suffer from psychosocial problems, lower academic achievement and premature death ${ }^{(5,6)}$.

The global rise in obesity prevalence, particularly in children, is too rapid to be explained by changes in genetic structure; therefore environmental factors are assumed to play an important role in the development of 
obesity risk behaviours ${ }^{(7)}$. High availability of highenergy foods along with limited opportunities to be physically active, the so-called 'obesogenic environment', is typical for Western European societies and is likely to promote excessive weight gain ${ }^{(8)}$.

It has been shown that non-Western immigrants living in Western societies are more often overweight and obese than non-Westerners who live in their country of origin ${ }^{(9)}$. A recent study conducted among schoolchildren in Europe showed that immigrant adolescents are more often overweight than native adolescents ${ }^{(10)}$. The non-Western immigrant population (e.g. from Turkey, Morocco, Surinam, Antillean, Iraq and Iran) forms the majority of migrants in the Netherlands ${ }^{(11)}$. Kleiser et al. ${ }^{(12)}$ showed considerable differences in dietary habits between young persons coming from different origins living in the Netherlands. Previous studies showed that the overweight prevalence of Turkish children living in the Netherlands is high with gender differences (in boys 23\%, in girls 30\%) and that unfavourable energy balance-related behaviours (EBRB), i.e. food intake, physical activity (PA) and sedentary behaviour, are common in this group ${ }^{(13-15)}$. Participation in PA has a strong socio-economic and ethnic gradient, with children of low socio-economic status or from an ethnic minority being less likely to participate in regular PA than their more advantaged counterparts ${ }^{(16,17)}$. Dutch children living in socio-economically deprived areas seem to have the lowest levels of PA and the highest rates of overweight $^{(18,19)}$. Considering such a large Turkish immigrant population (i.e. $21 \%$ of the non-Western immigrants), it is important to know their health behaviours and obesity and overweight patterns in order to reduce health inequalities. Exploring the potential influences of migration on weight status and related risk factors is possible by comparing immigrant adolescents with their peers living in Turkey. To the best of our knowledge, there is no study that has compared weight status and related risk behaviours of Turkish immigrant adolescents and their nonmigrant compatriots in Turkey. The current study aimed to compare the EBRB, measures of body composition and prevalence of overweight/obesity between Turkish adolescents in Turkey and Turkish immigrant adolescents in the Netherlands.

\section{Methods}

In the current study we used cross-sectional data from one Turkish survey and six Dutch studies with data on weight status and EBRB. All studies are compatible with regard to the study population, measures of body composition and assessed EBRB.

\section{Participants}

For the Turkish data set (TR-TR), a total of 915 (49\% girls) adolescents were randomly selected from healthy primary-school children living in Ankara, Turkey. For the Dutch data (TR-NL), a total of 433 (51\% girls) adolescents from primary and secondary schools from six different studies were included: i.e. DOiT ( $n$ 32), DOiT AGAIN ( $n$ 38), ENDORSE ( $n$ 106), ENERGY ( $n$ 24), JUMP-In ( $n$ 158) and Pro-Children $(n 75)^{(20-24)}$. From these Dutch data sets adolescents with a Turkish ethnicity (i.e. at least one of the parents was born in Turkey) were selected for data analysis. Data were collected in DOiT in 2003, DOiT AGAIN in 2010, ENDORSE in 2005/2006, ENERGY in 2011, JUMP-In in 2006, Pro-Children in 2003 and the Turkish survey in 2010.

Participation of adolescents in these different studies was voluntary. The response rates for both studies were similar, as for the TR-NL studies it varied from $79 \%$ to $90 \%$ and for the TR-TR study it was $82 \%$. Adolescents and their parents were informed about the objectives and methods of the studies and signed an informed consent form. The relevant ethical medical committees approved each study protocol. The inclusion criteria for the TR-TR study were to be healthy and to attend school regularly. In the TR-TR study the adolescents were recruited from three different public schools and had similar socio-economic levels.

\section{Antbropometric measurements}

Body weight $(\mathrm{kg})$, body height $(\mathrm{cm})$, waist and hip circumferences $(\mathrm{cm})$, and triceps, biceps, suprailiac and subscapular skinfold thicknesses $(\mathrm{mm})$ were measured using standard measurement protocols. There were some differences with regard to the measurement techniques between included studies, e.g. measuring children's body weight with clothes (ENDORSE) or in underwear (JUMPIn, DOiT, DOiT AGAIN), repeating the measurements two times (ENERGY, DOiT) or having only one measurement (JUMP-In). The detailed measurement techniques of each study are described in the online supplementary material.

BMI was calculated as body weight $(\mathrm{kg})$ divided by body height squared $\left(\mathrm{m}^{2}\right)$. Skinfold thickness measurements were summed. The definitions of overweight and obesity were based on age- and gender-specific BMI cutoff points for children and adolescents published by Cole et $a{ }^{(25)}$. Waist:hip ratio was calculated by dividing waist circumference by hip circumference.

\section{Energy balance-related behaviours}

EBRB included in the current study were: soft drink and fruit juice consumption $(\mathrm{ml} / \mathrm{d})$, fruit and vegetable $(\mathrm{F} \& \mathrm{~V})$ intake $(\mathrm{g} / \mathrm{d})$, breakfast frequency, daily PA ( $\mathrm{min}$ ) and screen time (min). Information on EBRB was collected by self-report questionnaires in all studies. Table 1 shows the available data on EBRB per study. The specific questions used in each study are provided in the online supplementary material.

\section{Soft drink and fruit juice consumption}

In Turkey and the Dutch Pro-Children study, data on soft drink and fruit juice consumption were collected using a 
Table 1 Available study variables by database per country

\begin{tabular}{|c|c|c|c|c|c|c|c|}
\hline & \multicolumn{6}{|c|}{ TR-NL ( $n$ 433) } & \multirow[b]{2}{*}{$\begin{array}{l}\text { TR-TP } \\
\text { (n } 915\end{array}$} \\
\hline & $\begin{array}{l}\text { DOiT } \\
(n 32)\end{array}$ & $\begin{array}{l}\text { DOIT AGAIN } \\
(n 38)\end{array}$ & $\begin{array}{l}\text { Jump-In } \\
\text { (n 158) }\end{array}$ & $\begin{array}{l}\text { ENERGY } \\
(n 24)\end{array}$ & $\begin{array}{l}\text { Pro-Children } \\
\quad(n 75)\end{array}$ & $\begin{array}{l}\text { ENDORSE } \\
(n 106)\end{array}$ & \\
\hline Body weight & 32 & - & 156 & 22 & - & 99 & 915 \\
\hline Body height & 32 & - & 156 & 22 & - & 98 & 915 \\
\hline Waist circumference & 32 & - & 156 & 22 & - & 99 & 508 \\
\hline Hip circumference & 32 & - & 156 & - & - & - & 508 \\
\hline Skinfold thickness & 31 & - & - & - & - & - & 508 \\
\hline TV watching & 31 & 37 & - & 21 & 75 & 104 & 508 \\
\hline Computer use & 30 & 37 & - & 22 & 72 & 105 & 508 \\
\hline Total physical activity & 32 & 35 & - & 24 & 73 & 103 & 508 \\
\hline Soft drink consumption & 20 & 36 & - & 23 & - & 97 & 915 \\
\hline Fruit juice consumption & 26 & 36 & - & 24 & 75 & - & 915 \\
\hline Fruit intake & - & - & - & - & 75 & - & 915 \\
\hline Vegetable intake & - & - & - & - & 75 & - & 915 \\
\hline Breakfast consumption & - & 37 & - & 23 & - & 15 & 915 \\
\hline
\end{tabular}

TR-NL, adolescents from Turkish immigrant ethnicity living in the Netherlands; TR-TR, Turkish adolescents in Turkey; TV, television.

$24 \mathrm{~h}$ dietary recall, i.e. amount of consumption on the day prior to the day of investigation. In the other studies data were collected by an FFQ recalling the frequency of consumption per a usual week and the amount per day, the same as in a Belgian study ${ }^{(26)}$. Consumption of soft drinks and fruit juice was summed to calculate total sugarcontaining beverage (SCB) consumption. SCB consumption of more than $5000 \mathrm{ml} / \mathrm{d}$ was considered as unrealistic and excluded from further data analysis.

\section{Fruit and vegetable intake}

The data on F\&V intake were collected only in two studies, i.e. the TR-TR study and the Pro-Children study. In Turkey and in the Dutch Pro-Children study, data on F\&V intake were collected using a $24 \mathrm{~h}$ dietary recall, i.e. number of portions consumed on the day prior to the day of investigation.

\section{Breakfast frequency}

In both Turkey and the Netherlands, breakfast frequency per week was assessed. In the Netherlands breakfast consumption was assessed separately for weekdays and weekend days. The variable was dichotomized as having breakfast every day or not.

\section{Physical activity}

In Turkey, PA recalls for three consecutive days were taken. The average time spent walking per day was calculated by the recalls. In the Dutch data sets, time spent in PA was collected differently in the different studies. The Pro-Children study recalled the duration of exercising in leisure time by one question; other studies assessed the frequency and time spent in active commuting to school, sports club activities and unorganized sport activities (DOiT, DOiT AGAIN, ENERGY); and in ENDORSE the frequency and duration of sport activities engaged in during the past week were recalled (see online supplementary material).

\section{Total screen time}

Total screen time was calculated as sum of television (TV) viewing time and computer time and expressed in minutes per day. In both countries the frequency (in a usual week) and duration of TV watching and computer use per day were recalled. In Turkey, ENDORSE, ENERGY and DOiT AGAIN, playing electronic games was specifically mentioned in the question as an example of computer use.

\section{Data analysis}

All data analyses were carried out using the statistical software package SPSS version $15 \cdot 0$. We investigated the effect of country, gender and their interaction due to earlier evidence on gender differences in weight status ${ }^{(13)}$. In the case of significant effects of gender by country interaction, the results were reported separately for girls and boys. We applied pairwise comparisons according to gender and country using ANCOVA to test group differences in EBRB. In ANCOVA the differences between groups were tested adjusting for covariates. Differences between groups in categorical outcomes (overweight status, daily breakfast consumption) were tested with logistic regression analyses.

Due to the variation in applied measurement methods in the different Dutch studies, we added a variable 'study cohort' as a covariate in the ANCOVA models (when there was more than one Dutch study per outcome). Furthermore, age and body height (when waist circumference was an outcome) were added as covariates in the ANCOVA models, both in the total group and also in gender group analyses. Gender was added as a covariate in the total group analyses. The level of significance was set as $P<0 \cdot 05$.

\section{Results}

In total, 469 boys and 446 girls living in Turkey (TR-TR) aged between 11 and 14 years (mean age $13 \cdot 1$ (SD $0 \cdot 8$ ) years) 
Table 2 Gender- and country-specific age and anthropometric characteristics of Dutch-Turkish (TR-NL) and Turkish-Turkish (TR-TR) adolescents

\begin{tabular}{|c|c|c|c|c|c|c|c|c|c|c|c|c|}
\hline & \multicolumn{4}{|c|}{ Boys } & \multicolumn{4}{|c|}{ Girls } & \multicolumn{4}{|c|}{ Total } \\
\hline & \multicolumn{2}{|c|}{ TR-NL ( $n$ 211) } & \multicolumn{2}{|c|}{ TR-TR ( $n$ 469) } & \multicolumn{2}{|c|}{ TR-NL ( $n$ 222) } & \multicolumn{2}{|c|}{ TR-TR ( $n$ 446) } & \multicolumn{2}{|c|}{ TR-NL ( $n$ 433) } & \multicolumn{2}{|c|}{ TR-TR ( $n$ 915) } \\
\hline & Mean & SD & Mean & SD & Mean & SD & Mean & SD & Mean & SD & Mean & SD \\
\hline Age (years) & $11 \cdot 7^{\star}$ & $1 \cdot 3$ & $13 \cdot 2$ & $0 \cdot 8$ & $11 \cdot 6^{*}$ & $1 \cdot 2$ & $13 \cdot 0$ & 0.8 & $11 \cdot 7^{*}$ & $1 \cdot 3$ & $13 \cdot 1$ & $0 \cdot 8$ \\
\hline Body weight (kg) & $48 \cdot 9^{*}$ & $13 \cdot 9$ & $47 \cdot 4$ & $9 \cdot 6$ & $50 \cdot 5^{\star}$ & $12 \cdot 6$ & $46 \cdot 6$ & $8 \cdot 4$ & $49 \cdot 7^{\star}$ & $13 \cdot 3$ & $47 \cdot 0$ & $9 \cdot 1$ \\
\hline Body height (cm) & $152 \cdot 5^{\star}$ & $10 \cdot 7$ & $154 \cdot 4$ & $8 \cdot 8$ & $152 \cdot 8^{*}$ & $8 \cdot 8$ & $152 \cdot 0$ & $9 \cdot 0$ & $152 \cdot 6^{*}$ & $9 \cdot 8$ & $153 \cdot 2$ & $9 \cdot 0$ \\
\hline BMI $\left(\mathrm{kg} / \mathrm{m}^{2}\right)$ & $20 \cdot 7^{*}$ & $3 \cdot 8$ & $19 \cdot 8$ & $3 \cdot 3$ & $21 \cdot 4^{*}$ & $4 \cdot 0$ & $20 \cdot 2$ & $3 \cdot 7$ & $21 \cdot 1^{*}$ & 3.9 & $20 \cdot 0$ & $3 \cdot 5$ \\
\hline Overweight (\%) & \multirow{2}{*}{\multicolumn{2}{|c|}{29}} & \multicolumn{2}{|c|}{25} & \multicolumn{2}{|c|}{$32^{*}$} & \multicolumn{2}{|c|}{26} & \multicolumn{2}{|c|}{$31^{*}$} & \multicolumn{2}{|c|}{26} \\
\hline Obese $(\%)$ & & & \multicolumn{2}{|c|}{5} & \multicolumn{2}{|c|}{$9^{*}$} & \multicolumn{2}{|c|}{7} & \multicolumn{2}{|c|}{$9^{\star}$} & \multicolumn{2}{|c|}{6} \\
\hline Waist circumference $(\mathrm{cm})$ & $72 \cdot 2$ & $10 \cdot 7$ & $71 \cdot 6$ & $9 \cdot 5$ & $72 \cdot 2^{\star}$ & $9 \cdot 8$ & $71 \cdot 0$ & $8 \cdot 8$ & $72 \cdot 2^{*}$ & $10 \cdot 3$ & $71 \cdot 3$ & $9 \cdot 2$ \\
\hline Hip circumference $(\mathrm{cm})$ & $80 \cdot 2^{*}$ & $7 \cdot 7$ & $88 \cdot 3$ & $7 \cdot 5$ & $84 \cdot 5$ & $8 \cdot 8$ & $89 \cdot 3$ & $7 \cdot 3$ & $82 \cdot 3^{*}$ & $8 \cdot 5$ & $88 \cdot 7$ & $7 \cdot 4$ \\
\hline Waist:hip ratio & 0.86 & 0.05 & $0 \cdot 81$ & 0.06 & 0.82 & 0.05 & 0.79 & 0.06 & 0.84 & 0.05 & $0 \cdot 80$ & 0.06 \\
\hline Triceps skinfold (mm) & $13 \cdot 6^{*}$ & $5 \cdot 1$ & $17 \cdot 3$ & $6 \cdot 6$ & $18 \cdot 1$ & $7 \cdot 4$ & $18 \cdot 2$ & $6 \cdot 4$ & $15 \cdot 5$ & $6 \cdot 5$ & $17 \cdot 7$ & $6 \cdot 5$ \\
\hline Biceps skinfold (mm) & $8 \cdot 8$ & $4 \cdot 1$ & $9 \cdot 6$ & $4 \cdot 9$ & $10 \cdot 9$ & $6 \cdot 2$ & $9 \cdot 7$ & $5 \cdot 1$ & $9 \cdot 7$ & $5 \cdot 1$ & $9 \cdot 7$ & $5 \cdot 0$ \\
\hline Subscapular skinfold (mm) & 11.9 & $7 \cdot 3$ & $12 \cdot 8$ & $5 \cdot 6$ & $18 \cdot 3^{*}$ & $13 \cdot 2$ & $13 \cdot 8$ & $5 \cdot 5$ & $14 \cdot 5$ & $10 \cdot 5$ & $13 \cdot 2$ & $5 \cdot 6$ \\
\hline Suprailiac skinfold (mm) & $19 \cdot 0^{*}$ & $11 \cdot 3$ & $12 \cdot 9$ & $6 \cdot 6$ & $21 \cdot 1^{*}$ & $11 \cdot 7$ & $13 \cdot 4$ & $6 \cdot 5$ & $19 \cdot 8^{*}$ & $11 \cdot 3$ & $13 \cdot 1$ & $6 \cdot 6$ \\
\hline Sum of skinfolds (mm) & $51 \cdot 9$ & $26 \cdot 4$ & $52 \cdot 6$ & $21 \cdot 3$ & $68 \cdot 4^{*}$ & $37 \cdot 4$ & $55 \cdot 1$ & $20 \cdot 8$ & $59 \cdot 2$ & $32 \cdot 3$ & $53 \cdot 7$ & $21 \cdot 1$ \\
\hline
\end{tabular}

Data are presented as mean and standard deviation unless otherwise stated. Data analyses were adjusted for age (all except age) and cohort (all except skinfold measures, due to having one cohort per country for this outcome), height (only for waist circumference and waist:height ratio) and gender (only for the total group comparison). ANCOVA and logistic regression (for overweight and obese percentages) were used for testing the differences between countries.

*Mean values were significantly different from those of the TR-TR group: $P<0.05$

and 211 boys and 222 girls with at least one parent born in Turkey, living in the Netherlands (TR-NL) and aged between 10 and 14 years (mean age $11 \cdot 7$ (SD 1.3) years) were included. This age difference between the study samples was statistically significant (Table 2).

\section{Measures of body composition and overweight/ obesity prevalence}

Mean age-adjusted body weight, BMI, waist circumference and suprailiac skinfold thickness were significantly higher in the TR-NL group than in the TR-TR group (Table 2), while the mean age-adjusted body height and hip circumference were significantly higher in the TR-TR group compared with the TR-NL group (all $P<0 \cdot 05$ ). Overweight and obesity were more prevalent in adolescents living in the Netherlands (31\% and 9\%, respectively) than in adolescents living in Turkey $(26 \%$ and $6 \%$, respectively; $P<0.05$; Table 2). Stratification by gender resulted in similar differences between the TR-TR and TR-NL groups, except that the difference in hip circumference and triceps skinfold thickness was smaller and not significant among girls, while differences in subscapular skinfold, sum of skinfolds, waist circumference, overweight and obesity were smaller and not significant in boys (Table 2).

\section{Energy balance-related behaviours}

\section{Dietary behaviours}

Daily average SCB consumption was approximately ten times higher in TR-NL than TR-TR adolescents (TR-NL: 1173 (sD 769) ml/d, TR-TR: 115 (sD 211) ml/d; $P<0 \cdot 05$ ). The total F\&V intake was significantly higher in TR-TR than TR-NL adolescents (TR-TR: 647 (SD 409) g/d, TR-NL: 295 (sD 293) g/d; $P<0 \cdot 05$ ). The proportion of adolescents reporting to eat breakfast daily was similar in both groups (TR-NL: 52\%, TR-TR: 54\%). Stratified analysis by gender yielded similar results, except the difference in fruit intake was smaller and not significant among boys (Table 3 ).

\section{Physical activity}

Table 3 shows the average PA levels. Total PA time was higher in the TR-NL than the TR-TR group (TR-NL: 61 (SD 91) $\mathrm{min} / \mathrm{d}$, TR-TR: 27 (sD 30) $\mathrm{min} / \mathrm{d} ; P<0 \cdot 05)$. Stratified analysis by gender gave similar results.

\section{Screen time}

Total self-reported screen time was significantly lower in the TR-NL adolescents (253 (sD 167) $\mathrm{min} / \mathrm{d}$ ) compared with the TR-TR adolescents (467 (sD 123) $\mathrm{min} / \mathrm{d} ; P<0 \cdot 05$ ). Computer time was also significantly lower in TR-NL adolescents than TR-TR adolescents, whereas TV time was significantly higher in TR-NL adolescents $(P<0 \cdot 05$; Table 3). Stratified analysis by gender yielded similar results, except the difference in TV time was not significant among boys.

\section{Discussion}

The present study examined differences in measures of body composition, overweight/obesity status and a selection of EBRB between Turkish adolescents living in Turkey and Turkish immigrant adolescents living in the Netherlands. Overweight and obesity rates were significantly higher among Turkish adolescents living in the Netherlands (TR-NL) compared with their peers living in Turkey (TR-TR). The TR-NL adolescents reported significantly higher SCB consumption and lower F\&V intake. 
Table 3 Energy balance-related behaviours of Dutch-Turkish (TR-NL) and Turkish-Turkish (TR-TR) adolescents

\begin{tabular}{|c|c|c|c|c|c|c|c|c|c|c|c|c|}
\hline & \multicolumn{4}{|c|}{ Boys } & \multicolumn{4}{|c|}{ Girls } & \multicolumn{4}{|c|}{ Total } \\
\hline & \multicolumn{2}{|c|}{ TR-NL ( $n$ 211) } & \multicolumn{2}{|c|}{ TR-TR ( $n$ 469) } & \multicolumn{2}{|c|}{ TR-NL ( $n$ 222) } & \multicolumn{2}{|c|}{ TR-TR ( $n$ 446) } & \multicolumn{2}{|c|}{ TR-NL ( $n$ 433) } & \multicolumn{2}{|c|}{ TR-TR ( $n$ 915) } \\
\hline & Mean & SD & Mean & SD & Mean & SD & Mean & SD & Mean & SD & Mean & SD \\
\hline TV watching $(\mathrm{min} / \mathrm{d})$ & $150 \cdot 8$ & $109 \cdot 8$ & $142 \cdot 0$ & $91 \cdot 7$ & $159 \cdot 6^{\star}$ & $121 \cdot 1$ & $123 \cdot 5$ & $86 \cdot 7$ & $155 \cdot 3^{\star}$ & $115 \cdot 6$ & $134 \cdot 0$ & $89 \cdot 9$ \\
\hline Computer use $(\mathrm{min} / \mathrm{d})$ & $109 \cdot 1^{*}$ & $89 \cdot 6$ & $315 \cdot 0$ & $148 \cdot 7$ & $89 \cdot 6^{*}$ & $94 \cdot 3$ & $359 \cdot 5$ & $164 \cdot 9$ & $99 \cdot 4^{*}$ & $92 \cdot 3$ & $334 \cdot 3$ & $157 \cdot 4$ \\
\hline Total screen time $(\mathrm{min} / \mathrm{d})$ & $257 \cdot 6^{\star}$ & $160 \cdot 0$ & $454 \cdot 9$ & $116 \cdot 0$ & $248 \cdot 5^{\star}$ & $174 \cdot 6$ & $483 \cdot 0$ & $129 \cdot 6$ & $252 \cdot 9^{\star}$ & $167 \cdot 3$ & $467 \cdot 1$ & $122 \cdot 8$ \\
\hline Total PAt $(\mathrm{min} / \mathrm{d})$ & $79 \cdot 8^{\star}$ & $102 \cdot 8$ & $29 \cdot 2$ & $30 \cdot 2$ & $43 \cdot 1^{*}$ & $73 \cdot 7$ & $24 \cdot 8$ & $28 \cdot 6$ & $61 \cdot 0^{*}$ & $90 \cdot 8$ & $27 \cdot 3$ & $29 \cdot 6$ \\
\hline Soft drink consumption $(\mathrm{ml} / \mathrm{d})$ & $858 \cdot 3^{*}$ & $675 \cdot 4$ & $69 \cdot 1$ & $134 \cdot 5$ & $728 \cdot 3^{*}$ & $596 \cdot 3$ & $96 \cdot 2$ & $166 \cdot 9$ & $790 \cdot 3^{\star}$ & $636 \cdot 8$ & $82 \cdot 3$ & $151 \cdot 7$ \\
\hline Fruit juice consumption $(\mathrm{ml} / \mathrm{d})$ & $458 \cdot 9^{\star}$ & $417 \cdot 7$ & $25 \cdot 1$ & $68 \cdot 6$ & $395 \cdot 4^{\star}$ & $366 \cdot 2$ & $41 \cdot 0$ & $96 \cdot 0$ & $425 \cdot 8^{*}$ & $391 \cdot 7$ & 32.9 & $83 \cdot 4$ \\
\hline Total SCB intake $(\mathrm{ml} / \mathrm{d})$ & $1210 \cdot 8^{\star}$ & $845 \cdot 8$ & $94 \cdot 2$ & $184 \cdot 0$ & $1140 \cdot 9^{\star}$ & $706 \cdot 1$ & $137 \cdot 2$ & $235 \cdot 1$ & $1173 \cdot 1^{*}$ & $769 \cdot 1$ & $115 \cdot 2$ & $211 \cdot 4$ \\
\hline Fruit intake $(g / d)$ & $187 \cdot 9$ & $207 \cdot 0$ & $291 \cdot 7$ & $307 \cdot 3$ & $208 \cdot 5^{\star}$ & $243 \cdot 6$ & $360 \cdot 6$ & $330 \cdot 1$ & $199 \cdot 2^{*}$ & $226 \cdot 5$ & $325 \cdot 3$ & $320 \cdot 3$ \\
\hline Vegetable intake $(\mathrm{g} / \mathrm{d})$ & $99 \cdot 7^{*}$ & $112 \cdot 9$ & $313 \cdot 8$ & $215 \cdot 7$ & $92 \cdot 1^{*}$ & $106 \cdot 1$ & $329 \cdot 7$ & $214 \cdot 7$ & $95 \cdot 5^{\star}$ & $108 \cdot 6$ & $321 \cdot 6$ & $215 \cdot 2$ \\
\hline Total F\&V intake $(\mathrm{g} / \mathrm{d})$ & $287 \cdot 6^{\star}$ & $252 \cdot 8$ & $605 \cdot 5$ & 391.5 & $300 \cdot 6^{*}$ & $325 \cdot 7$ & $690 \cdot 2$ & $422 \cdot 8$ & $294 \cdot 7^{\star}$ & 293.0 & $646 \cdot 8$ & $409 \cdot 0$ \\
\hline Daily breakfast consumption‡ (\%) & 55 & & & & 49 & & & & 52 & & & 4 \\
\hline
\end{tabular}

TV, television; PA, physical activity; SCB, sugar-containing beverage; F\&V, fruit and vegetable.

Data are presented as mean and standard deviation unless otherwise stated. Data analyses were adjusted for age (for all), cohort (except for fruit, vegetable and total F\&V intakes, due to having one cohort per country for these outcomes) and gender (only for the total group comparison). ANCOVA and logistic regression (for daily breakfast consumption) were used for testing the differences between countries. Total SCB consumption variable was log-transformed in the ANCOVA model due to non-normal distribution of the residuals.

*Mean values were significantly different from those of the TR-TR group: $P<0.05$.

tWalking, biking, sports.

‡Having breakfast every day.

Furthermore, TR-NL adolescents reported higher PA levels but less screen time and computer use.

The mechanisms underlying the high prevalence of obesity are complex and multifactorial ${ }^{(27)}$. Many immigrant groups of non-Western origin living in Western societies have high levels of overweight/obesity ${ }^{(18,28)}$. The high rates of overweight and obesity among immigrant adolescents may reflect a response to their obesogenic environment; migrants' behaviour is likely to be influenced by their socio-cultural and physical environment ${ }^{(29)}$. On the one hand, the physical environment is likely to be the first element changing the behaviour of immigrants and changes in availability and accessibility of facilities might influence participation in $\mathrm{EBRB}^{(27)}$. On the other hand, changes in social and cultural norms also influence food intake and PA patterns of immigrants. Acculturation - the process of coherence to the host culture that occurs with migration - influences diet, PA and preferences of body size of immigrants ${ }^{(9)}$. This acculturation can positively influence health and related behaviours. However, behavioural change of migrants is not always in a positive way and not in the same direction for all behaviours (either healthy or less healthy changes) ${ }^{(9)}$. The act of immigration may also affect health, since it may be stressful with negative psychosocial impacts. These influences may explain our finding that Turkish immigrant adolescents in the Netherlands reported less favourable dietary habits, reflected in drinking significantly more SCB and eating less F\&V, compared with Turkish adolescents living in Turkey. A recent study including eight European countries showed that Dutch adolescents reported the highest quantities of soft drink consumption $^{(30)}$. Thus, Turkish immigrant adolescents in the Netherlands may be influenced by the social norm and peer modelling of consuming much SCB or the high availability of SCB in the Netherlands. Regarding the differences in F\&V intake, in Turkey it is traditional in families to serve fruits after dinner. Among OECD (Organisation for Economic Co-operation and Development) countries, numbers from Turkey indicate very high levels of F\&V consumption ${ }^{(31)}$ and this may reflect the dietary habits of Turkish families living in Turkey. A recent study indicated that among Dutch children daily average $F \& V$ intake was $224 \mathrm{~g}$ in girls and $218 \mathrm{~g}$ in boys $^{(32)}$. This shows that Turkish immigrant children report higher F\&V intake than Dutch children but lower F\&V intake than Turkish children in Turkey.

Turkish adolescents living in the Netherlands reported significantly lower screen times. Among the TR-TR group computer time (average $334 \mathrm{~min} / \mathrm{d}$ ) was significantly higher than in TR-NL adolescents (average $99 \mathrm{~min} / \mathrm{d}$ ), while TV time (average $134 \mathrm{~min} / \mathrm{d}$ in TR-TR) was significantly higher in TR-NL adolescents (average $155 \mathrm{~min} / \mathrm{d}$ ). Computer use in Turkey became widespread especially after the 1990s and has continued to increase rapidly since the beginning of the $2010 \mathrm{~s}^{(33)}$. A recent study showed that most high-school adolescents (67\%) use Internet cafés to play computer games, chat and surf the Internet; boys prefer going to such places more than girls in Turkey ${ }^{(34)}$. Gathering to play multi-player games in Internet cafés is way of socializing with friends and increases the computer time of Turkish children ${ }^{(35)}$. Cultural and/or parental rules for watching TV as well as the accessibility of other competing/concurrent media like computers may influence the amount of time young people spend watching $\mathrm{TV}^{(36)}$. Results of the ENERGY study suggest that non-native Dutch children spend more time in screen activities than natives $(157 \mathrm{~min} / \mathrm{d}$ in 
non-natives $v .118 \mathrm{~min} / \mathrm{d}$ in natives) ${ }^{(11)}$. This indicates that Turkish immigrant children report higher screen time than Dutch children but lower screen time than Turkish children in Turkey.

TR-TR adolescents reported significantly lower PA levels than TR-NL adolescents. A recent European study showed that Dutch adolescents are among the most active in Europe, especially with regard to active transportation $^{(30)}$. Adolescents may learn from others by observing, thus social support and social network from friends/ family and schools may influence being active ${ }^{(37)}$. Turkish immigrant adolescents in the Netherlands might be influenced by the high social norm of active transportation from/to school and the active transportation facilities (e.g. separate bicycle lanes) in the Netherlands. However, Dutch adolescents from Turkish ethnicity are still less active than native Dutch adolescents ${ }^{(13)}$

Our results confirmed that dietary, PA and sedentary habits are influenced by the physical environment and the social and cultural norms.

\section{Strengths and limitations}

This comparison study in a large sample of adolescents is unique. We had data from Turkish immigrant adolescents from several different cities in the Netherlands where the majority of the Turkish immigrant population lives, improving the generalizability of our findings. Furthermore, we had objectively measured anthropometric data. A weakness of the study is the different measurement instruments used, especially with regard to the selfreported measures. Differences between countries might partly be explained by the assessment methods. The differences in total PA measurement methods may cause underestimation of total PA in some cohorts. Furthermore, EBRB were assessed by self-report, thus suffering from recall bias and socially desirable answers. When interpreting findings it should be kept in mind that differences in the data collection period (i.e. Dutch surveys between 2003 and 2011, Turkish survey in 2010) may have influenced the results. For example, the result from current surveys, i.e. having significantly more screen time and F\&V consumption among TR-TR children, may reveal the ongoing behaviour change among children in the last years. Furthermore, results from the comparison of F\&V intake that is limited to two studies and skinfold thickness measurement that is sensitive for inter-observer variation need to be interpreted more carefully.

\section{Conclusion}

The important limitations mentioned above should be firmly kept in mind in drawing conclusions from the present study. In conclusion, Turkish immigrant adolescents in the Netherlands showed higher levels of overweight and distinct patterns of EBRB. The results further suggest that their dietary patterns were less favourable than their peers living in Turkey while their PA and screen time were more favourable. These findings suggest that Turkish immigrant adolescents in the Netherlands have an orientation towards their host culture, influenced by the socio-cultural environment they live in. This might result from the process of acculturation and integration into the Dutch culture. We should also encourage TR-NL children to consume more F\&V like their Turkish counterparts. Furthermore, TR-NL children should be encouraged to consume fewer soft drinks. To reduce disparities and health inequalities in the Netherlands, it is important to understand the social and physical environmental factors that contribute to overweight and obesity among immigrant adolescents.

\section{Acknowledgements}

Sources of funding: The contribution of G.A. was funded by the ERASMUS programme at Gazi University and M.Y. was funded by Wereld Kanker Onderzoek Fonds (WCRF NL 2008/65), as part of the World Cancer Research Fund international grant programme. M.M.v.S. was financially supported by a fellowship of the EMGO Institute for Health and Care Research. The DOiT intervention was funded by the Netherlands Heart Foundation (NHF-2000Z002). The Jump-In study was supported financially by the Public Health Fund and the Ministry of Health, Welfare and Sport. The ENERGY project was funded by the Seventh Framework Programme (CORDIS FP7) of the European Commission, HEALTH (FP7HEALTH-2007-B; grant agreement no. 223254) and was additionally supported by a grant from the Netherlands Organization for Health Research and Development (grant no. 50-50150-98-002). The contribution of the ProChildren study was funded by the Commission of the European Communities, specific RTD Programme 'The Quality of Life and Management of Living Resources' (QLK1-2001-00547). The ENDORSE study was funded by a grant from ZonMw, the Netherlands Organization for Health Research and Development (grant ID no. 2100.0103). The funders had no role in the design, analysis or writing of this article. Conflicts of interest: The authors declare that they have no conflicts of interest. Ethical approval: The DOiT, DOiT AGAIN, JUMP-In and ENERGY studies were approved by the Medical Ethics Committee of the VU University Medical Center. The ENDORSE and Pro-Children studies were approved by the Medical Ethics Committee of Erasmus University Medical Center. The Turkish study was approved by the Scientific Advisory Committee of Gazi University, Faculty of Health Sciences. Authors' contributions: A.S.S., S.J.t.V., J.B., A.O., J.S.B.d.M. and M.J.M.C. are the principal investigators of the included Dutch studies. N.S., N.A.-T., G.A., S.B., M.G.-K., E.K. and H.Y. designed the Turkish study and collected the data. G.A. and M.Y. analysed the data and 
wrote and revised the manuscript, All authors advised with results and conclusions, reviewed the manuscript and contributed at multiple revisions.

\section{Supplementary material}

To view supplementary material for this article, please visit http://dx.doi.org/10.1017/S1368980013003388

\section{References}

1. Cecchini M, Sassi F, Lauer JA et al. (2010) Tackling of unhealthy diets, physical inactivity, and obesity: health effects and cost-effectiveness. Lancet 376, 1775-1784.

2. Wiegand S, Bau AM \& Babitsch B (2011) Dietary interventions and social care for treating obesity in children. Bundesgesundheitsblatt Gesundheitsforschung Gesundheitsschutz 54, 533-540.

3. Lobstein $\mathrm{T}$, Baur L \& Uauy R; IASO International Obesity Task Force (2004) Obesity in children and young people: a crisis in public health. Obes Rev 5, Suppl. 1, 4-104.

4. Singh AS, Mulder C, Twisk JW et al. (2008) Tracking of childhood overweight into adulthood: a systematic review of the literature. Obes Rev 9, 474-488.

5. Singh GK, Yu SM, Siahpush M et al. (2008) High levels of physical inactivity and sedentary behaviors among US immigrant children and adolescents. Arch Pediatr Adolesc Med 162, 756-763.

6. Singh A, Uijtdewilligen L, Twisk JW et al. (2012) Physical activity and performance at school: a systematic review of the literature including a methodological quality assessment. Arch Pediatr Adolesc Med 166, 49-55.

7. Sahingoz SA \& Sanlier N (2011) Compliance with Mediterranean Diet Quality Index (KIDMED) and nutrition knowledge levels in adolescents. A case study from Turkey. Appetite 57, 272-277.

8. Nicolaou M, Benjelloun S, Stronks K et al. (2012) Influences on body weight of female Moroccan migrants in the Netherlands: a qualitative study. Health Place 18, 883-891.

9. Nicolaou M, Doak CM, van Dam RM et al. (2009) Cultural and social influences on food consumption in Dutch residents of Turkish and Moroccan origin: a qualitative study. J Nutr Educ Behav 41, 232-241.

10. Brug J, van Stralen MM, Chinapaw MJ et al. (2012) Differences in weight status and energy-balance related behaviours according to ethnic background among adolescents in seven countries in Europe: the ENERGY-project. Pediatr Obes 7, 399-411.

11. Brussaard JH, van Erp-Baart MA, Brants HA et al. (2001) Nutrition and health among migrants in The Netherlands. Public Health Nutr 4, 659-664.

12. Kleiser C, Mensink GB, Neuhauser H et al. (2010) Food intake of young people with a migration background living in Germany. Public Health Nutr 13, 324-330.

13. Fredriks AM, Buuren SV, Sing RAH et al. (2005) Alarming prevalences of overweight and obesity for children of Turkish, Moroccan and Dutch origin in The Netherlands according to international standards. Acta Paediatr 94, 496-498.

14. De Wilde JA, Van Dommelen P, Middelkoop BJ et al. (2009) Trends in overweight and obesity prevalence in Dutch, Turkish, Moroccan and Surinamese South Asian children in the Netherlands. Arch Dis Child 94, 795-800.

15. Van Vuuren L, Stegeman H, Van Dieren L et al. (2012) Zo gezond zijn Amsterdamse jongeren!: stadsrapport Amsterdamse Jeugdgezondheidsmonitor voortgezet onderwijs
2010-2011; GGD Amsterdam. Epidemiologie, Documentatie \& Gezondheidsbevordering. http://www.gezond.amsterdam.nl/ publish/pages/473214/zo_gezond_zijn_amsterdamse_jongeren.pdf (accessed November 2012).

16. Crespo CJ, Ainsworth BE, Keteyian SJ et al. (1999) Prevalence of physical inactivity and its relation to social class in US adults: results from the Third National Health and Nutrition Examination Survey, 1988-1994. Med Sci Sports Exerc 31, 1821-1827.

17. Lowry R, Kann L, Collins JL et al. (1996) The effect of socioeconomic status on chronic disease risk behaviors among US adolescents. JAMA 276, 792-797.

18. Zeijl E, Crone M, Wiefferink K et al. (2005) Kinderen in Nederland (Children in The Netherlands). Den Haag: Sociaal en Cultureel Planbureau.

19. De Vries SI, Bakker I, van Overbeek K et al. (2005) Kinderen in Prioriteitswijken: lichamelijke (in)activiteit en overgewicht (Children in Deprived City Areas: Physical (In)Activity and Overweight). Leiden: TNO Kwaliteit van Leven.

20. Singh AS, Chin A, Paw MJ et al. (2006) Design of the Dutch Obesity Intervention in Teenagers (NRG-DOiT): systematic development, implementation and evaluation of a school-based intervention aimed at the prevention of excessive weight gain in adolescents. BMC Public Health 6, 304 .

21. De Meij JS, Chinapaw MJ, Kremers SP et al. (2010) Promoting physical activity in children: the stepwise development of the primary school-based JUMP-In intervention applying the RE-AIM evaluation framework. $\mathrm{BrJ}$ Sports Med 44, 879-887.

22. van Stralen MM, te Velde SJ, Singh AS et al. (2011) EuropeaN Energy balance Research to prevent excessive weight Gain among Youth (ENERGY) project: design and methodology of the ENERGY cross-sectional survey. BMC Public Health 11, 65.

23. Anderson AS (2006) The Pro-Children Project - a crossnational approach to increasing fruits and vegetables in the next generation and onwards. Int J Behav Nutr Phys Act 3, 26.

24. Van der Horst K, Oenema A, Van de Looij-Jansen P et al. (2008) The ENDORSE study: research into environmental determinants of obesity related behaviors in Rotterdam schoolchildren. BMC Public Health 8, 142.

25. Cole TJ, Bellizzi MC, Flegal KM et al. (2000) Establishing a standard definition for child overweight and obesity worldwide: international survey. BMJ 320, 1240-1243.

26. Vereecken CA \& Maes L (2003) A Belgian study on the reliability and relative validity of the Health Behaviour in School-Aged Children food frequency questionnaire. Public Health Nutr 6, 581-588.

27. Swinburn B, Egger G \& Raza F (1999) Dissecting obesogenic environments: the development and application of a framework for identifying and prioritizing environmental interventions for obesity. Prev Med 29, 563-570.

28. Van den Berg G, Van Eijsden M, Vrijkotte TG et al. (2012) Socioeconomic inequalities in lipid and glucose metabolism in early childhood in a population-based cohort: the ABCD-Study. BMC Public Health 12, 591.

29. Kirchengast S \& Schober E (2006) To be an immigrant: a risk factor for developing overweight and obesity during childhood and adolescence? J Biosoc Sci 38, 695-705.

30. Brug J, van Stralen MM, Te Velde SJ et al. (2012) Differences in weight status and energy-balance related behaviors among schoolchildren across Europe: the ENERGY-project. PLoS One 7, e34742.

31. Srinivasan CS, Irz X \& Shankar B (2006) An assessment of the potential consumption impacts of WHO dietary norms in OECD countries. Food Policy 31, 53-77. 
32. Fischer C, Brug J, Tak NI et al. (2011) Differences in fruit and vegetable intake and their determinants among 11-year-old schoolchildren between 2003 and 2009. Int J Behav Nutr Phys Act 8, 141.

33. Turkish Statistical Institute (2010) The results of the 2010 research on the use of information technologies in the household. http://www.tuik.gov.tr/PreTablo.do?tb_id=60\& ust_id $=2$. (accessed November 2012).

34. Gurol M \& Sevindik T (2007) Profile of Internet cafe users in Turkey. Telematics Informatics 4, 59-68.
35. Karakus T, Inal Y \& Cagiltay K (2008) A descriptive study of Turkish high school students' game-playing characteristics and their considerations concerning the effects of games. Comput Hum Behav 24, 2520-2529.

36. Tahiroglu AY, Celik GG, Uzel M et al. (2008) Internet use among Turkish adolescents. Cyber Psychol Behav 11, 537-543.

37. Ståhl T, Rütten A, Nutbeam D et al. (2001) The importance of the social environment for physically active lifestyle results from an international study. Soc Sci Med 52, 1-10. 